\title{
Termination Analysis for Abductive General Logic Programs
}

\author{
Sofie Verbaeten* \\ Department of Computer Science, K.U.Leuven \\ Celestijnenlaan 200A, B-3001 Heverlee, Belgium \\ sofie.verbaeten@cs.kuleuven.ac.be
}

\section{Introduction}

Proving termination of programs is important in any approach to program development. In this paper, we address the termination behaviour of abductive general logic programs and queries. Abduction is a form of reasoning which, given a knowledge base and an observation $Q$, finds possible explanations of $Q$ in terms of a particular set of predicates, called the abducible predicates. In the context of logic programming, abductive procedures have been used for planning, knowledge assimilation and belief revision, database updating, reasoning in the context of temporal domains with uncertainty, ... (we refer to [7] for references to such works). In [7], Denecker and De Schreye present an abductive extension of SLDNF [1], called SLDNFA. We study termination of general logic programs executed under SLDNFA w.r.t. an arbitrary safe selection rule. We show that the termination conditions for SLDNF of Apt and Bezem [3], namely acyclicity of the program and boundedness of the query w.r.t. a level mapping, are not sufficient for ensuring termination of SLDNFA. In particular, these conditions do not prevent an SLDNFA-derivation from abducing an infinite number of abducible atoms. We propose a third, syntactical condition, namely abductive non recursivity of the program and query. This condition together with the acyclicity of the program and boundedness of the query are sufficient for proving termination of an SLDNFA-derivation using a safe selection rule. For definite programs and queries as well as for programs and queries without abducible predicates, the condition of abductive nonrecursivity is trivially satisfied. So, in these cases, termination of SLDNFA is implied by the acyclicity of the program and boundedness of the query. By the best of our knowledge, this is the first work on termination of an abductive procedure for general logic programs.

After a short section of preliminaries, we briefly recall in Section 3 the SLDNFA proof procedure as defined in [7]. In Section 4, we present a sufficient termination condition, extending the condition of [3], for SLDNFA executed under an arbitrary safe selection rule. We conclude in Section 5 . We refer to the full version of the paper, [8], for a formal definition of SLDNFA, a motivation of our choice for SLDNFA as proof procedure for abductive general programs (see also [7]), a detailed explanation of our termination condition, all the proofs and more examples. An extended version of this paper was accepted for publication in the proceedings of ICLP'99.

\footnotetext{
*Research Assistant of the Fund for Scientific Research - Flanders (Belgium)(F.W.O.).
} 


\section{Preliminaries}

We assume familiarity with the basic concepts of logic programming [2]. Throughout the paper, $P$ will denote a general logic program based on an alphabet $\Sigma$. With ground $\Sigma(P)$ we denote the set of $\Sigma$-ground instances of clauses of $P$. With $\Sigma^{p}$ we denote the set of predicate symbols of $\Sigma$. With $U_{P}$, resp. $B_{P}$, we denote the Herbrand Universe, resp. Herbrand Base, associated with ( $\Sigma$ underlying) $P$. With $\neg B_{P}$ we denote the set of negative ground literals $\left\{\neg A \mid A \in B_{P}\right\}$.

Let $p, q \in \Sigma^{p}$. We say that $p$ refers to $q$ in $P$ iff there is a clause in $P$ with $p$ in the head and $q$ occurring in the body. We say that $p$ depends on $q$ in $P$, and write $p \sqsupseteq q$, iff $(p, q)$ is in the reflexive, transitive closure of the relation refers to. We write $p \simeq q$ iff $p \sqsupseteq q$ and $q \sqsupseteq p$ ( $p$ and $q$ are mutually recursive or $p=q)$, and $p \sqsupset q$ iff $p \sqsupseteq q$ and $q \nsupseteq p$. If $L$ is a literal $p\left(t_{1}, \ldots, t_{n}\right)$ or $\neg p\left(t_{1}, \ldots, t_{n}\right)$, then we define $\operatorname{Rel}(L)=p$. With a signed predicate of a set $\Pi$ of predicate symbols, we mean $p$ or $\neg p$, where $p \in \Pi$. An atom of the form $p(\bar{t})$ will be called a $p$-atom.

\section{The SLDNFA Proof Procedure}

By the lack of space, we refer to $[8,7]$ for a formal definition of the SLDNFA proof procedure. In this section, we will give the main ideas together with an example.

An abductive logic program $P^{A}$ based on $\Sigma$ is a general program $P$ based on $\Sigma$ together with a subset $A$ of undefined predicates of $\Sigma^{p}$, called abducible. The other predicates in $\Sigma^{p} \backslash A$ are called nonabducible. An SLDNFA computation can be understood as a process of deriving formulas $\forall\left(Q_{0} \leftarrow \Phi\right)$, with $\leftarrow Q_{0}$ the initial query and the conjunction $\Phi$ composed of two types of unsolved queries:

- For any query $\leftarrow Q$ for which a derivation still needs to be computed (in the sequel, a positive $q u e r y$ ), $\Phi$ contains the open formula $Q$ (denoting the open conjunction of literals in $Q$ ).

- For any query $\leftarrow Q$ for which a failure tree still needs to be constructed (in the sequel, a negative query), $\Phi$ contains the open formula $\forall \bar{X} . \leftarrow Q$ with $\bar{X}$ a subset of the variables of $Q$.

SLDNFA selects unsolved positive or negative queries $\leftarrow Q$ and literals in $\leftarrow Q$ and rewrites these queries depending on the sort of selection. These rewrite operations can be interpreted as theorem proving steps on $\Phi$. Resolution is applied on nonabducible atoms selected in positive and negative queries, as in SLDNF. Negative literals are deleted from positive queries and added as negative queries and vice versa, just as in SLDNF. Abducible atoms in positive queries are never selected: they are treated as residual atoms. They are called abduced atoms. The process of computing an increasing set of residual abducible atoms can be understood as the incremental construction of a definition for the abducible predicates. Abducible atoms selected in negative queries are resolved with all the residual atoms in the positive queries.

Note that $\Phi$ contains two types of variables: free variables (universally quantified in front of $\left.\forall\left(Q_{0} \leftarrow \Phi\right)\right)$, in the sequel called positive variables, and variables universally quantified in a conjunct of $\Phi$, in the sequel called negative variables. This distinction plays a crucial role in SLDNFA. Whether variables in queries in a derivation are positive or negative depends on the way they are introduced in the derivation. The positive variables are either variables of the initial query $Q_{0}$ or are the variables of input program clauses used for resolution with positive queries. The negative variables are the variables of program clauses used for resolution with negative queries. The operations of unification and resolution in SLDNFA are modified to take the difference between 
positive and negative variables into account. In order to inform unification and resolution in SLDNFA about the logical nature of the variables, i.e. whether they are positive or negative, variables in a derivation have a superscript + or - . We give a small fault diagnosis problem of [7] to illustrate the SLDNFA proof procedure.

Example 3.1 A faulty lamp problem is caused by a broken lamp or by a power failure of a circuit without backup, that is, a loaded battery. The only circuit with battery is $c 1$; its battery is b1. A battery is unloaded iff one of its energy cells is dry. This is formalized in the program $P^{\left\{b r o k e n / 1, \text { power_failure } / 1, d r y_{-} \text {cell } / 1\right\}}$ :

$$
\begin{aligned}
& \operatorname{lamp}(l 1) \leftarrow \\
& \text { battery }(c 1, b 1) \leftarrow \\
& \text { faulty_lamp } \leftarrow \operatorname{lamp}(X) \text {, broken }(X) \\
& \text { faulty_lamp } \leftarrow \text { power_failure }(X), \neg \text { backup }(X) \\
& \text { backup }(X) \leftarrow \operatorname{battery}(X, Y), \neg \text { unloaded }(Y) \\
& \text { unloaded }(X) \leftarrow \text { dry_cell }(X)
\end{aligned}
$$

An SLDNFA-refutation for the query $\leftarrow$ faulty_lamp is shown in Fig. 1 . As a notational conve-

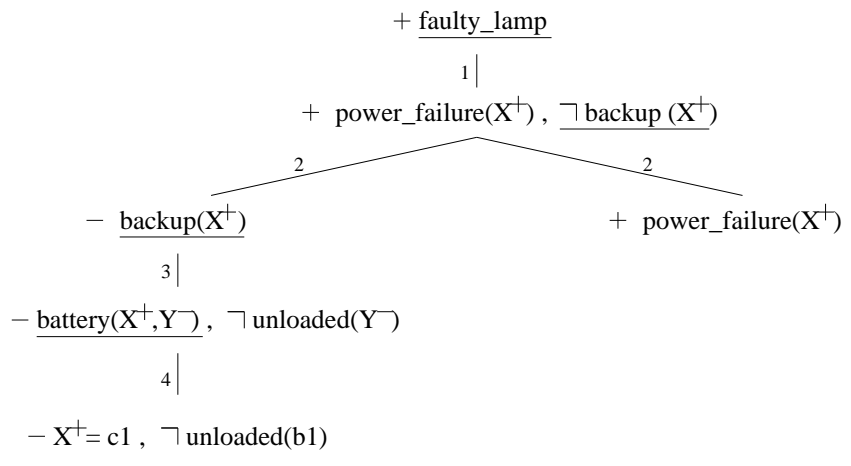

Figure 1: SLDNFA-refutation for $\leftarrow$ faulty_lamp.

nience, we prefix positive, resp. negative, queries with + , resp. -. The selected literal in a query is underlined and the arcs are numbered to indicate the sequence of computation steps.

In the first step, the atom faulty_lamp in the (positive) initial query is resolved with a program clause. In the second step, the selected negative atom $\neg$ backup $\left(X^{+}\right)$gives rise to two descendants: the negative query consisting of one atom backup $\left(X^{+}\right)$and the positive query obtained from the selected query by deleting $\neg$ backup $\left(X^{+}\right)$. This positive query contains one abducible atom power_failure $\left(X^{+}\right)$which will never be selected (as said before, abducible atoms in positive queries are treated as residual atoms). The negative query with the selected nonabducible atom backup $\left(X^{+}\right)$is resolved against all program clauses whose head unify with the selected atom. This introduces the negative variable $Y^{-}$. The same is done for the selected atom battery $\left(X^{+}, Y^{-}\right)$in the subsequent negative query. As can be seen in step 4, resolution of an atom in a negative query differs from the classical resolution by the fact that positive variables are never bound. Instead, disequality constraints are generated on them. A disequality constraint is an equality atom in a negative query, which belongs to the mgu of the preceding resolution step, and is of the form $X^{+}=t$, where $t$ is either a nonvariable term or another positive variable. Such an equality atom is also called irreducible. The answer that can be extracted from this refutation is that there is a power failure on a circuit $X^{+}$that is not $c 1$. 


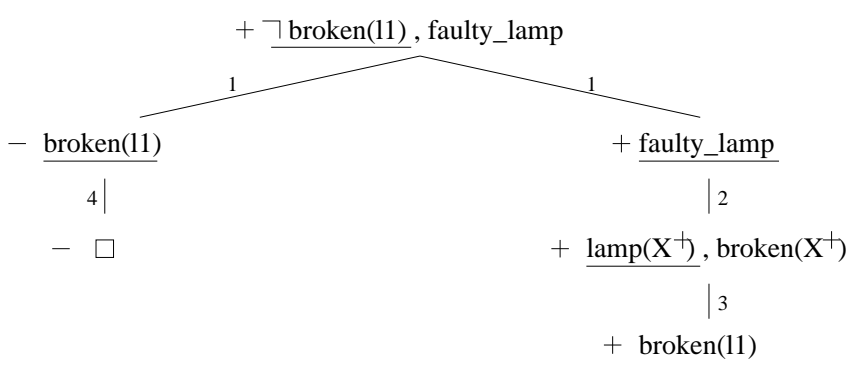

Figure 2: Finitely failed derivation for $\leftarrow \neg$ broken(l1), faulty lamp.

Fig. 2 shows a finitely failed SLDNFA-derivation for the query $\leftarrow \neg$ broken(l1), faulty_lamp. Note that in the 4th step, the abducible atom broken $(l 1)$ in the negative query is resolved with the abduced atom broken(l1) (obtained in the 3th step). Since this resolution succeeds and the empty negative query is obtained, the derivation fails.

In Example 4.4, we will prove that all SLDNFA-derivations for $\leftarrow$ faulty_lamp are finite. In fact, we can prove that for every query, all SLDNFA-derivations in $P^{\left\{\text {broken } / 1, p_{\left.\text {ower-failure } / 1, d r y \_c e l l / 1\right\}}\right.}$ are finite (this is discussed at the end of Section 4).

In the following section, we study termination under SLDNFA-execution w.r.t. an arbitrary selection rule. However, we put 3 natural conditions on the selection in SLDNFA, namely: (1) no abducible atom is selected in a positive query (by definition of SLDNFA, they are treated as residual atoms); (2) no negative literal containing negative variables is selected in a negative query (i.e. the selection is $s a f e^{1}$ ); (3) no irreducible equality atom is selected in a negative query (this is a reasonable condition, since the descendant in that case would be identical to the negative query). For the rest of this paper, we consider SLDNFA-derivations using a selection rule satisfying the above conditions.

\section{SLDNFA-Termination}

Let $P^{A}$ be an abductive logic program and $\leftarrow Q$ be a query, both based on the alphabet $\Sigma$.

Definition 4.1 (SLDNFA-terminating program w.r.t. a query)

$P^{A}$ is SLDNFA-terminating w.r.t. $\leftarrow Q$ iff all SLDNFA-derivations of $\leftarrow Q$ in $P^{A}$ are finite.

As in most termination analyses for logic programs (see [6] for a survey), our termination condition relies on the concepts of level mapping and boundedness.

\section{Definition 4.2 (level mapping, boundedness)}

$A$ level mapping for $P^{A}$ is a function $||:. B_{P} \cup \neg B_{P} \rightarrow \mathbb{N}$, with $|\neg B|=|B|$ for all $B \in B_{P}$. A literal $L$ is called bounded w.r.t. |.| iff |.| is bounded on the set of $\Sigma$-ground instances of $L$. A query $\leftarrow Q$ is called bounded w.r.t. |.| iff all its literals are.

We introduce the notion of semi-acyclic program, which is equivalent ${ }^{2}$ to the notion of acyclic program [3]. However, as noted in [4] in the case of definite programs, the notion of semi-acyclicity

\footnotetext{
${ }^{1}$ SLDNFA offers a partial solution to the negation floundering problem: positive variables may appear in selected negative literals. However, SLDNFA does not offer a solution for the treatment of negative variables in positive queries, hence this weak safety condition on the selection.

${ }^{2}$ The proof of this equivalence is similar to the proof of the equivalence of these notions in the case of definite programs (called semi-recurrency and recurrency [5] in that case), see [4].
} 
allows more natural level mappings and provides means for constructing modular termination proofs.

Definition 4.3 (semi-acyclic program) Let $|$.$| be a level mapping for P^{A}$. $P^{A}$ is semi-acyclic w.r.t. |.| iff $\forall H \leftarrow L_{1}, \ldots, L_{n} \in G_{\text {round }}\left(P^{A}\right)$ and $\forall i \in\{1, \ldots, n\}$ :

$$
\begin{aligned}
& |H|>\left|L_{i}\right| \quad \text { if } \operatorname{Rel}(H) \simeq \operatorname{Rel}\left(L_{i}\right), \\
& |H| \geq\left|L_{i}\right| \quad \text { if } \operatorname{Rel}(H) \sqsupset \operatorname{Rel}\left(L_{i}\right) .
\end{aligned}
$$

$P^{A}$ is semi-acyclic iff there exists a level mapping |.| such that $P^{A}$ is semi-acyclic w.r.t. |.|.

Note that, since abducible predicates do not occur in the head of a clause, in the search for a level mapping such that $P^{A}$ is semi-acyclic, it can be safely assumed that abducible literals are assigned the value 0 .

In [3] it was proven that for an acyclic general program $P$ and a bounded query $\leftarrow Q$, all SLDNF-derivations of $\leftarrow Q$ in $P$ are finite. In the following example we show that this is not the case for SLDNFA.

Example 4.1 Consider the program $P^{\{p / 1\}}$ with query $\leftarrow \neg r, p(X)$.

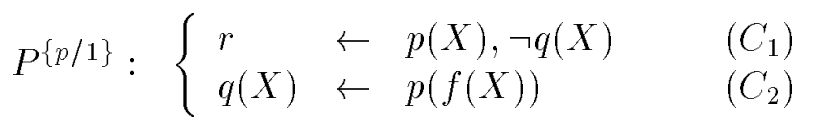

Consider the following level mapping $|$.$| for P^{\{p / 1\}}:|r|=2,|q(t)|=1$ and $|p(t)|=0$ for all $t \in U_{P}$. Then, $P\{p / 1\}$ is (semi-)acyclic w.r.t. |.| and the query $\leftarrow \neg r, p(X)$ is bounded w.r.t. |.|.

An SLDNFA-derivation for $\leftarrow \neg r, p(X)$ in $P^{\{p / 1\}}$ is shown in Fig. 3 . Note that the derivation is

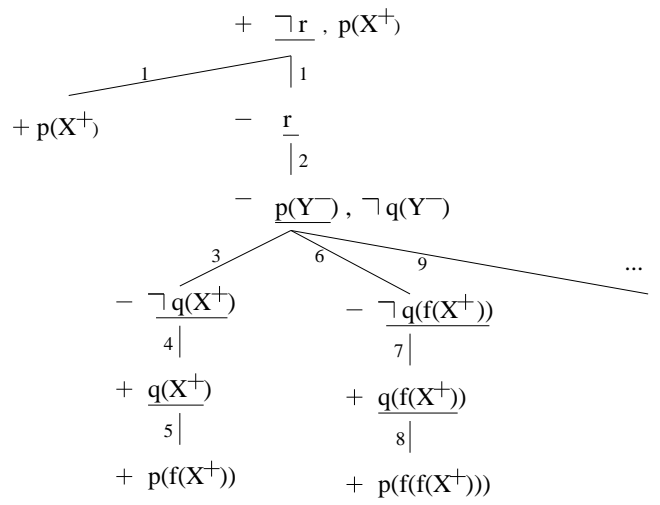

Figure 3: SLDNFA-derivation for $\leftarrow \neg r, p(X)$ in $P^{\{p / 1\}}$.

not finite, since there is an infinitely branching (negative) node ${ }^{3}$.

As the above example suggests, an additional condition (besides (semi-)acyclicity of the program and boundedness of the query) is needed in order that the program is SLDNFA-terminating w.r.t. the query. In particular, we need a condition which prevents an SLDNFA-derivation from being

\footnotetext{
${ }^{3}$ Resolution of the selected abducible atom $p\left(Y^{-}\right)$in the negative query $-p\left(Y^{-}\right), \neg q\left(Y^{-}\right)$, with the abduced atom $p\left(X^{+}\right)$(step 3) leads to the abduction of $p\left(f\left(X^{+}\right)\right.$) (step 5), which in turn has to be resolved with $p\left(Y^{-}\right)$(step 6), leading to the abduction of $p\left(f\left(f\left(X^{+}\right)\right)\right.$) (step 8$)$, which in turn ...
} 
infinitely branching in a node. Note that a derivation can only be infinitely branching in a node which contains a negative query with a selected abducible $p$-atom and there are an infinite number of abduced $p$-atoms in positive queries. Hence, the additional condition should ensure that, for every derivation of $\leftarrow Q$ in $P^{A}$, the set of abduced atoms in the derivation is finite. Note that for propositional programs and queries, this condition is satisfied. So, in the propositional case, SLDNFA-termination is implied by the (semi-)acyclicity of the program and boundedness of the query. Concerning the predicate case, we introduce a syntactical condition on a program and query, called abductive nonrecursivity (Definition 4.5), which, together with the (semi-)acyclicity of the program and boundedness of the query, implies termination of SLDNFA (Theorem 4.1). We introduce the necessary concepts.

Definition 4.4 (reductions and paths) Let $P^{A}$ be a program and $\leftarrow Q$ be a query. Let start be a new (i.e. not occurring in $\Sigma^{p}$ ) predicate symbol of arity 0 . We define reductions between elements of the form $* s p$, where $* \in\{+,-\}$ and sp is a signed predicate symbol of $\Sigma^{p} \cup\{$ start $\}$. The reductions will be denoted with an arrow " $\rightarrow$ ", indexed with the query $Q$, a clause of $P^{A}$, or the symbols.

- Suppose $\leftarrow Q=\leftarrow \ldots, p(\bar{t}), \ldots, \neg q(\bar{s}), \ldots$. Then we have the following $\rightarrow_{Q}$-reductions:

$$
+ \text { start } \rightarrow_{Q}+p \quad+\text { start } \rightarrow_{Q}+\neg q
$$

- Let $C=h(\bar{u}) \leftarrow \ldots, p(\bar{t}), \ldots, \neg q(\bar{s}), \ldots$ be a clause of $P^{A}$. Then we have the following $\rightarrow{ }_{C}$-reductions:

$$
+h \rightarrow_{C}+p \quad+h \rightarrow_{C}+\neg q \quad-h \rightarrow_{C}-p \quad-h \rightarrow_{C}-\neg q
$$

- For all predicate symbols $p \in \Sigma^{p}$, we have the following $\rightarrow_{s^{-} \text {-reductions: }}$

$$
+\neg p \rightarrow_{s}-p \quad-\neg p \rightarrow_{s}+p
$$

A reduction sequence of the form + start $\rightarrow_{Q} \cdot\left(\rightarrow_{s}\right) . \rightarrow_{C} \cdot\left(\rightarrow_{s}\right) . \rightarrow_{C^{\prime}} \ldots . .\left(\rightarrow_{s}\right) . \rightarrow_{C^{\prime \prime}} \ldots$, where $C, C^{\prime}, C^{\prime \prime}$ are clauses of $P^{A}$, is called a path from $\leftarrow Q$ in $P^{A}$.

If there is a subpath (possibly of length 0 ) in a path from $\leftarrow Q$ in $P^{A}$ starting in $*$ sp and ending in $*^{\prime} s q$, where $*, *^{\prime} \in\{+,-\}$ and $s p, s q$ are signed predicates of $\Sigma^{p} \cup\{$ start $\}$, then we denote this with $* s p \rightarrow^{Q, P, s} *$ 'sq. If in this subpath from $* s p$ to $*$ 'sq, no reduction of the sort $\rightarrow_{s}$ occurs, then we denote this with $* s p \rightarrow{ }^{Q, P} *^{\prime} s q$ (note that in this case $*=*^{\prime}$ ).

An element of the form $* s p$, with $* \in\{+,-\}$ and $s p$ a signed predicate (as it appears in the above definition) can be seen as an abstraction of a positive or negative (depending on *) query which contains a positive or negative (depending on the sign of $s p$ ) literal with as predicate symbol the predicate of $s p$. Branches in an SLDNFA-derivation of $\leftarrow Q$ in $P^{A}$ can then, in some way, be abstracted by paths from $\leftarrow Q$ in $P^{A}$. We give an example.

Example 4.2 Recall the program $P^{\{p / 1\}}$ and query $\leftarrow Q=\leftarrow \neg r, p(X)$ of Example 4.1. We have e.g. the following paths from $\leftarrow \neg r, p(X)$ in $P^{\{p / 1\}}:+$ start $\rightarrow_{Q}+\neg r \rightarrow_{s}-r \rightarrow_{C_{1}}-p$ and + start $\rightarrow_{Q}+\neg r \rightarrow_{s}-r \rightarrow_{C_{1}}-\neg q \rightarrow_{s}+q \rightarrow_{C_{2}}+p$. Notice that these paths can be found as the branches 1-2 and 1-2-3-4-5 (or 1-2-6-7-8) resp. in the SLDNFA-derivation of Fig. 3. It follows e.g. that $-r \rightarrow^{Q, P}-p$ and $-r \rightarrow^{Q, P, s}+p$. 
Definition 4.5 (abductive recursive program and query) Let $P^{A}$ be a program and $\leftarrow Q$ be a query. We call $P^{A} \cup\{\leftarrow Q\}$ abductive recursive iff there are abducible predicates $p_{1}, \ldots, p_{n} \in A$ and predicates $q_{1}, \ldots, q_{n} \in \Sigma^{p} \backslash(A \cup\{=\}), n \geq 1$, such that

$$
\begin{array}{llllllllll}
-q_{1} & \rightarrow^{Q, P} & -p_{1} & -q_{2} & \rightarrow^{Q, P} & -p_{2} & & -q_{n} & \rightarrow^{Q, P} & -p_{n} \\
-q_{1} & \rightarrow^{Q, P, s} & +p_{2} & -q_{2} & \rightarrow^{Q, P, s} & +p_{3} & \cdots & -q_{n} & \rightarrow^{Q, P, s} & +p_{1}
\end{array}
$$

We call $P^{A} \cup\{\leftarrow Q\}$ abductive nonrecursive iff $P^{A} \cup\{\leftarrow Q\}$ is not abductive recursive.

In [8], the meaning of the notion of abductive recursive program and query in terms of SLDNFAderivations is given. We give the main idea here. Let $T$ be an SLDNFA-derivation. A positive, resp. negative, query $N$ in $T$ is prefixed with + , resp. - . If a query $N$ in $T$ contains the atom $p(\bar{t})$, then this will be denoted as $N(p)$. If a node $N$ is an ancestor of a node $M$ in $T$, then this will be denoted as $N \sim M$. With this notation, we have the following. Suppose the query $\leftarrow Q$ has an SLDNFA-derivation $T$ in $P^{A}$ and $T$ has nodes $-N_{1}\left(p_{1}\right),+M_{1}\left(p_{2}\right),-N_{2}\left(p_{2}\right),+M_{2}\left(p_{3}\right), \ldots,-N_{n}\left(p_{n}\right)$, $+M_{n}\left(p_{1}\right)$ with $n \geq 1$ and $p_{1}, p_{2}, \ldots, p_{n} \in A$, such that $-N_{1}\left(p_{1}\right) \sim+M_{1}\left(p_{2}\right),-N_{2}\left(p_{2}\right) \sim+M_{2}\left(p_{3}\right)$, $\ldots,-N_{n}\left(p_{n}\right) \sim+M_{n}\left(p_{1}\right)$, then $P \cup\{\leftarrow Q\}$ is abductive recursive.

Example 4.3 Recall the program $P^{\{p / 1\}}$ and query $\leftarrow Q=\leftarrow \neg r, p(X)$ of Example 4.1. We see that for the SLDNFA-derivation of $\leftarrow \neg r, p(X)$ in $P^{\{p / 1\}}$ of Fig. 3, we have that $-N_{1}(p) \sim+M_{1}(p)$ with $-N_{1}=-p\left(Y^{-}\right), \neg q\left(Y^{-}\right)$and $+M_{1}=+p\left(f\left(X^{+}\right)\right)$. Hence, by the discussion above, $P^{\{p / 1\}} \cup\{\leftarrow$ $\neg r, p(X)\}$ is abductive recursive. Indeed, this follows from Definition 4.5 , since $-r \rightarrow^{Q, P}-p$ and $-r \rightarrow^{Q, P, s}+p$ (see Example 4.2).

Theorem 4.1 (main) Let $P^{A}$ be a semi-acyclic program w.r.t. a level mapping |.|, and let $\leftarrow Q$ be a bounded query w.r.t. |.|. Suppose that $P^{A} \cup\{\leftarrow Q\}$ is abductive nonrecursive. Then, $P^{A}$ is SLDNFA-terminating w.r.t. $\leftarrow Q$.

Example 4.4 Recall the program and query $\leftarrow$ faulty_lamp of Example 3.1. Let |.| be the following level mapping: $\mid$ broken $(t)|=|$ power_failure $(t)|=| \operatorname{dry}_{\text {_cell }}(t)|=0,| \operatorname{lamp}(t)|=|$ battery $(t, s) \mid$ $=|\operatorname{unloaded}(t)|=1, \mid$ backup $(t) \mid=2$ and $\mid$ faulty_lamp $\mid=3$, with $t, s \in U_{P} . P$ is (semi-) acyclic w.r.t. |.| and the query $\leftarrow$ faulty_lamp is bounded w.r.t.|.|. One can easily verify that $P \cup\{\leftarrow$ faulty_lamp $\}$ is abductive nonrecursive, so, $P$ SLDNFA-terminates w.r.t. $\leftarrow$ faulty_lamp.

Example 4.5 Recall the program $P^{\{p / 1\}}$ of Example 4.1. As we already noted in Example 4.3, $P^{\{p / 1\}} \cup\{\leftarrow \neg r, p(X)\}$ is abductive recursive. Consider next the query $\leftarrow r$. Note that $P\{p / 1\} \cup\{\leftarrow$ $r\}$ is abductive nonrecursive. By the fact that $P^{\{p / 1\}}$ is (semi-)acyclic and $\leftarrow r$ is bounded w.r.t. the level mapping proposed in Example 4.1, we have that $P^{\{p / 1\}}$ SLDNFA-terminates w.r.t. $\leftarrow r$.

Note that, in case $A=\emptyset$, a program $P^{\{\}}$and query $\leftarrow Q$ are trivially abductive nonrecursive. So, the termination condition of Theorem 4.1 boils down to the termination condition of Apt and Bezem [3] for SLDNF in that case. Note also that a definite program and query are trivially abductive nonrecursive. So, also in that case, termination is ensured by the (semi-)acyclicity of the program $^{4}$ and boundedness of the query.

The notion of abductive recursivity of Definition 4.5 depends on the program and the query in question. We refer to Example 4.5 where a program and two queries are given such that the program and first query are abductive recursive (and do not SLDNFA-terminate) whereas the program and

\footnotetext{
${ }^{4}$ Note that for definite programs, the notion of (semi-)acyclicity is called (semi-)recurrency [5, 4].
} 
second query are abductive nonrecursive (and do SLDNFA-terminate). We refer to [8] where we formulate a condition on the program only, which we also call abductive nonrecursivity (but now with no reference to a particular query), such that the program $P^{A}$ is abductive nonrecursive iff for all queries $\leftarrow Q, P^{A} \cup\{\leftarrow Q\}$ is abductive nonrecursive. As a corollary to the main Theorem 4.1, we then prove in [8] that: If $P^{A}$ is a semi-acyclic program w.r.t. a level mapping $|$.$| and P^{A}$ is abductive nonrecursive, then, for all bounded queries $\leftarrow Q$ w.r.t.|.|, $P^{A}$ is SLDNFA-terminating w.r.t. $\leftarrow Q$. By means of this termination condition, we can prove that the program of Example 3.1 SLDNFA-terminates w.r.t. all queries (see [8]).

\section{Conclusion and Future Work}

We presented an extension of the methods of Apt and Bezem [3] for proving termination of general logic programs executed under SLDNF, to the case of abductive general logic programs executed under SLDNFA [7]. Whereas the conditions of Apt and Bezem (i.e. acyclicity of the program and boundedness of the query) are also sufficient for proving termination of SLDNFA in the case of definite programs and queries, in the propositional case, as well as in the case of the empty set of abducible predicates, their conditions are not sufficient in general for proving SLDNFA-termination. We proposed a third, syntactical condition, namely abductive nonrecursivity of the program and query, which, together with the acyclicity of the program and boundedness of the query, implies termination of SLDNFA. By the best of our knowledge, this is the first work on termination of an abductive procedure for general logic programs.

Our termination condition (Theorem 4.1) is sufficient but not necessary. In particular, there are (semi-)acyclic programs and bounded queries which SLDNFA-terminate, but which are abductive recursive. It remains a topic for future work to refine the notion of abductive recursivity and to find a characterisation of SLDNFA-termination. Another topic for future research is to study termination of SLDNFA w.r.t. a particular selection rule.

\section{References}

[1] K. R. Apt and K. Doets. A new definition of SLDNF-resolution. Journal of Logic Programming, 18:177190, 1994.

[2] K.R. Apt. Logic programming. In J. van Leeuwen, editor, Handbook of Theoretical Computer Science, Vol. B. Elsevier Science Publishers, 1990.

[3] K.R. Apt and M. Bezem. Acyclic programs. New Generation Computing, 9:335-363, 1991.

[4] K.R. Apt and D. Pedreschi. Modular termination proofs for logic and pure Prolog programs. In Advances in Logic Programming Theory, pages 183-229. Oxford University Press, 1994.

[5] M. Bezem. Strong termination of logic programs. Journal of Logic Programming, 15:79-97, 1993.

[6] D. De Schreye and S. Decorte. Termination of logic programs: the never-ending story. Journal of Logic Programming, 19 \& 20:199-260, 1994.

[7] M. Denecker and D. De Schreye. SLDNFA: an abductive procedure for abductive logic programs. Journal of Logic Programming, 34(2):111-167, 1998.

[8] S. Verbaeten. Termination analysis for abductive general logic programs. Technical report, Department of Computer Science, K.U.Leuven, 1999. Available at http://www.cs.kuleuven.ac.be/ sofie. 